\title{
HOMOLOGY OF A FIBRATION OVER AN ASPHERICAL SPACE
}

\author{
YASUTOSHI NOMURA
}

\begin{abstract}
The aim of this paper is to establish, for a fibration over a space of type $(Q, 1)$, an exact sequence involving the homology homomorphisms induced by the projection. Specializing to the case where the total space is aspherical, our sequence allows us to add some extra terms to the Eckmann-Stammbach exact sequence for a group extension with simple integer coefficient.
\end{abstract}

1. Statement of the result. All spaces, maps and homotopies in this paper are assumed to be base-pointed and all spaces are assumed to have the homotopy type of a CW complex.

Let $F_{\rightarrow}^{i} E_{\rightarrow}^{p} B$ be a Hurewicz fibration with path-connected $F$ and $B$. Through the operation $\mu$ of $\Omega B$, the space of loops on $B$, on $F$ (see [1, §3]), $\pi_{1}(B)$ operates on $H_{*}(F)$; thus, $H_{*}(F)$ is a module over $Z \pi_{1}(B)$, the integral group ring of $\pi_{1}(B)$. Let $Q$ denote $\pi_{1}(B)$ and we write $\operatorname{Tor}_{k}^{Q}$ for $\operatorname{Tor}_{k}^{Z Q}$. In the next section we prove

THEOREM 1.1. Suppose that $\pi_{k}(B)=0$ for $1<k<n-m+1$ and that $H_{q}(F)=0$ for $0<q<m$ and $m<q<n$, where $1 \leqq m<n$. Then there exists an exact sequence

$$
\begin{aligned}
H_{n+1}(E) & \stackrel{p_{*}}{\longrightarrow} H_{n+1}(B) \longrightarrow R \longrightarrow H_{n}(E) \\
& \stackrel{p_{*}}{\longrightarrow} H_{n}(B) \longrightarrow \operatorname{Tor}_{n-m-1}^{Q}\left(H_{m}(F), Z\right) \\
& \longrightarrow H_{n-1}(E) \longrightarrow \cdots \longrightarrow H_{m+1}(B) \\
& \longrightarrow H_{m}(F) \otimes Q \\
& \longrightarrow \longrightarrow H_{m}(E) \\
& \stackrel{p_{*}}{\longrightarrow} H_{m}(B) \longrightarrow 0,
\end{aligned}
$$

in which $R$ is an abelian group such that the row and column are exact in the

Received by the editors September 13, 1971.

AMS 1970 subject classifications. Primary 55F20; Seçondary 55F05, 18H10, $20 \mathrm{~J} 05$.

Key words and phrases. Homology group, fibration, aspherical space, homology of a group, group extension, Whitney join, cofibre.

(c) American Mathematical Society 1972 
following commutative diagram

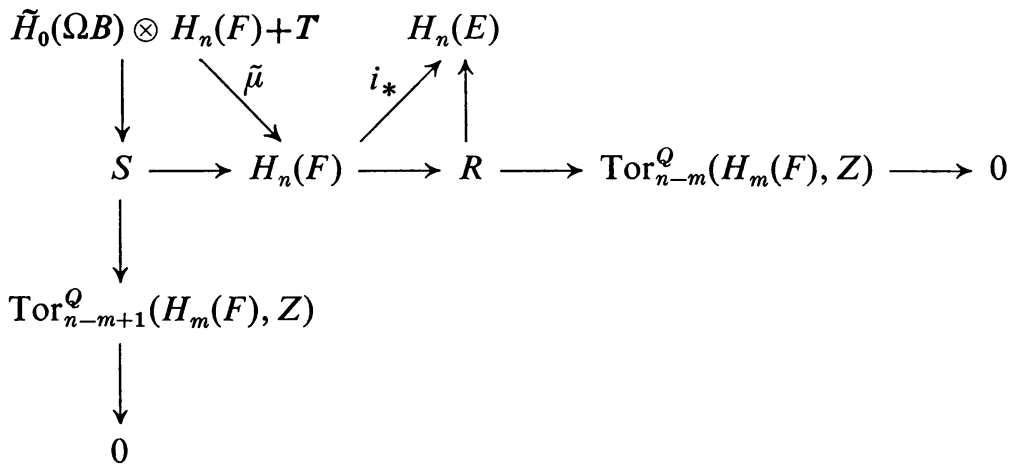

where $\tilde{\mu}$ is induced by $\mu$ and $T=H_{n-m}(\Omega B) \otimes H_{m}(F)$.

COROLlARY 1.2. Under the assumption of Theorem 1.1, there exists an exact sequence

$$
\begin{aligned}
H_{n+1}(B) & \rightarrow \operatorname{Tor}_{n-m}^{Q}\left(H_{m}(F), Z\right) \rightarrow H_{n}(E) / i_{*} H_{n}(F) \\
& \rightarrow H_{n}(B) \rightarrow \cdots \rightarrow H_{m}(B) \rightarrow 0 .
\end{aligned}
$$

Now let

$$
1 \rightarrow N \rightarrow G \rightarrow Q \rightarrow 1
$$

be a group extension. As usual, $G$ acts on $N$ by conjugation, which induces the action of $Q$ on $H_{*}(N)$, the coefficient of which is the additive group of integers $Z$ with trivial operators. In the preceding situation let $B$ and $E$ be aspherical of type $(Q, 1)$ and $(G, 1)$ respectively, and let $p$ induce the homomorphism $G \rightarrow Q$ in (1). Then $F$ is aspherical of type $(N, 1)$. Since the topological action of $\pi_{1}(B)$ on $H_{*}(F)$ coincides with the above one of $Q$ on $H_{*}(N)$ by [5, Corollary 1.4], Theorem 1.1 implies

Corollary 1.3. Suppose that $H_{q}(N)=0$ for $0<q<m$ and $m<q<n$, where $1 \leqq m<n$. Then there exists an abelian group $R^{\prime}$ making the following sequences exact:

$$
\begin{aligned}
H_{n+1}(G) & \rightarrow H_{n+1}(Q) \rightarrow R^{\prime} \rightarrow H_{n}(G) \rightarrow H_{n}(Q) \\
& \rightarrow \operatorname{Tor}_{n-m-1}^{Q}\left(H_{m}(N), Z\right) \rightarrow H_{n-1}(G) \\
& \rightarrow \cdots \rightarrow H_{m}(N) \otimes_{Q} Z \rightarrow H_{m}(G) \rightarrow H_{m}(Q) \rightarrow 0
\end{aligned}
$$

and

$$
H_{n}(N) \rightarrow R^{\prime} \rightarrow \operatorname{Tor}_{n-m}^{Q}\left(H_{m}(N), Z\right) \rightarrow 0 .
$$

In particular, if $H_{n-m}\left(Q ; H_{m}(N)\right)=0$ (e.g. if $Q$ acts trivially on $H_{m}(N)$, $H_{n-m}(Q)=0$ and $\left.\operatorname{Tor}\left(H_{n-m-1}(Q), H_{m}(N)\right)=0\right)$, then there is an exact 
sequence

$H_{n}(N) \rightarrow H_{n}(G) \rightarrow H_{n}(Q) \rightarrow \operatorname{Tor}_{n-m-1}^{Q}\left(H_{m}(N), Z\right) \rightarrow \cdots \rightarrow H_{m}(Q) \rightarrow 0$.

For $m=1$ the first half of Corollary 1.3 extends the exact sequence obtained by Eckmann and Stammbach [2, Theorem 4.3, (VII')] (cf. [7, sequence (A)]). For $n=m+1$, the second half is just the second part of Theorem 1 of [5]. Note that Corollary 1.2, applied to (1), yields a generalization of Theorem 2.1 of Ganea [3].

2. Proof of Theorem 1.1. As usual, $S$ stands for the suspension functor and the cofibre of a map $f: X \rightarrow Y$ is denoted by $C_{f}$. Let $P B$ denote the space of paths in $B$ emanating from basepoint and let

$$
p_{k}: E_{k} \rightarrow B, \quad E_{k}=P B \oplus \cdots \oplus P B \oplus E \quad(k \geqq 1)
$$

be the Whitney join of $p_{0}=p: E \rightarrow B$ and $k$ path-fibrations $P B \rightarrow B$ (see [4]). The fibre $F_{k}$ of $p_{k}$ is the join

$$
\underset{(k \text { factors })}{\Omega B *}(\Omega B .
$$

There are canonical inclusions $j_{k}: E_{k-1} \rightarrow E_{k}$ satisfying $p_{k} j_{k}=p_{k-1}$, which induce the maps $c_{k}: C_{p_{k-1}} \rightarrow C_{p_{k}}$.

LEMMA 2.1. The cofibre of $j_{k}$ is of the same homotopy type as $S F_{k-1}$.

Proof. Since $E_{k}$ is the double mapping cylinder of $P B \leftarrow K_{k} \rightarrow E_{k-1}$ (cf. [4]), where

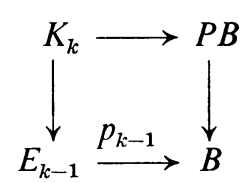

is a pullback square, hence $K_{k}$ is homotopy equivalent to $F_{k-1}$, we see that $E_{k}$ is homotopically equivalent to the cofibre of $F_{k-1} \rightarrow E_{k-1}$. Thus, from the Puppe sequence of $F_{k-1} \rightarrow E_{k-1}$ the assertion follows.

LEMMA 2.2 .

$$
\begin{aligned}
& H_{q}\left(F_{k-1}\right) \cong 0 \text { if } 0<q<m+k-1 \text { or } m+k-1<q<n+k-1 \text {, } \\
& \cong \tilde{H}_{0}(\Omega B) \otimes \cdots \otimes \tilde{H}_{0}(\Omega B) \otimes H_{m}(F) \text { if } q=m+k-1 \text {. }
\end{aligned}
$$

Proof. Since $\pi_{i}(\Omega B)=0$ for $1 \leqq i<n-m$ and since each path component of $\Omega B$ is in 1-1 correspondence with an element of $\pi_{1}(B)$, it follows readily that $H_{q}(\Omega B)=0$ for $1 \leqq q<n-m$. Therefore the lemma is a consequence of the Künneth theorem. 
LemMA 2.3. The cofibre of $c_{k}$ is homotopy equivalent to $S^{2} F_{k-1}$, hence $c_{k^{*}}: H_{q}\left(C_{p_{k-1}}\right) \rightarrow H_{q}\left(C_{p_{k}}\right)$ is

bijective for $q<m+k$ or $m+k+1<q<n+k$;

monic for $q=m+k+1$;

epic for $q=m+k$ or $n+k$.

Proof. Let $C B$ denote the cone over $B$. Introduce the following commutative diagram

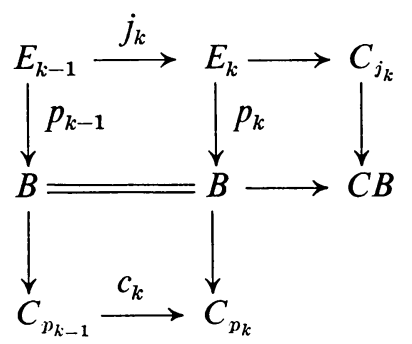

By Lemma 2.1, $C_{j_{k}}$ is homotopy equivalent to $S F_{k-1}$, so that it follows from the $3 \times 3$ lemma (cf. [4, Lemma 1.2]) that the cofibre of $c_{k}$ is homotopy equivalent to $S^{2} F_{k-1}$, hence the first assertion. This, together with Lemma 2.2, implies the second assertion.

Now let $I$ denote the augmentation ideal of the integral group ring $Z Q$, that is, the kernel of $\varepsilon: Z Q \rightarrow Z$; thus, the sequence

$$
0 \longrightarrow I \longrightarrow Z Q \stackrel{\varepsilon}{\longrightarrow} Z \longrightarrow 0
$$

is exact and $I$ is $Z$-free. Note that $H_{0}(\Omega B) \cong Z Q, \tilde{H}_{0}(\Omega B) \cong I$. $Q$ acts on $I \otimes \cdots \otimes I$ ( $k$ factors) through $x\left(y_{1} \otimes \cdots \otimes y_{k}\right)=x y_{1} \otimes \cdots \otimes x y_{k}, x \in Q, y_{i} \in I$.

The following is a consequence of the construction due to Schmid [6, p. 31].

LEMma 2.4. Let $M$ be a $Z Q$-module. Then $H_{k}(Q, M)=\operatorname{Tor}_{k}^{Q}(Z, M)$ is isomorphic to the kernel of the homomorphism

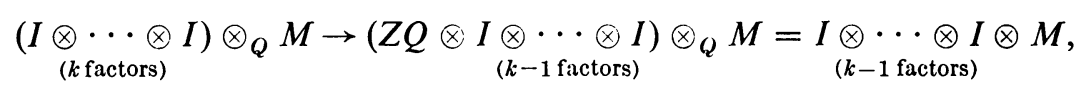

that is, to the homology of the 0-sequence

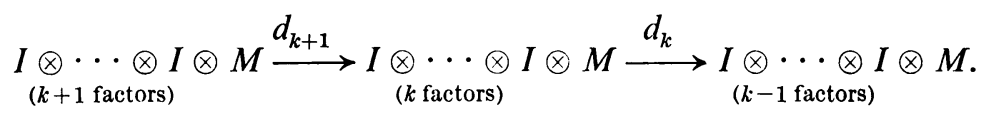

where $d_{k}$ is given by $d_{k}\left((\omega-1) \otimes x_{2} \otimes \cdots \otimes x_{k} \otimes m\right)=x_{2} \omega^{-1} \otimes \cdots \otimes x_{k} \omega^{-1} \otimes$ $\omega m-x_{2} \otimes \cdots \otimes x_{k} \otimes m$ for $\omega \in Q, x_{i} \in I, m \in M$.

Let $\sigma_{k}: S\left(\Omega B * F_{k-1}\right)=S F_{k} \rightarrow C_{p_{k}}$ be the map defined in Lemma 1.1 of [5] for the fibration $p_{k}$ and let $H(\mu): \Omega B * F_{k-1} \rightarrow S F_{k-1}$ be the map obtained 
from the action $\mu: \Omega B \times F_{k-1} \rightarrow F_{k-1}$ by the Hopf construction. Let $i_{k}: F_{k} \rightarrow E_{k}$ denote the fibre inclusion. Consider the homotopy commutative diagram

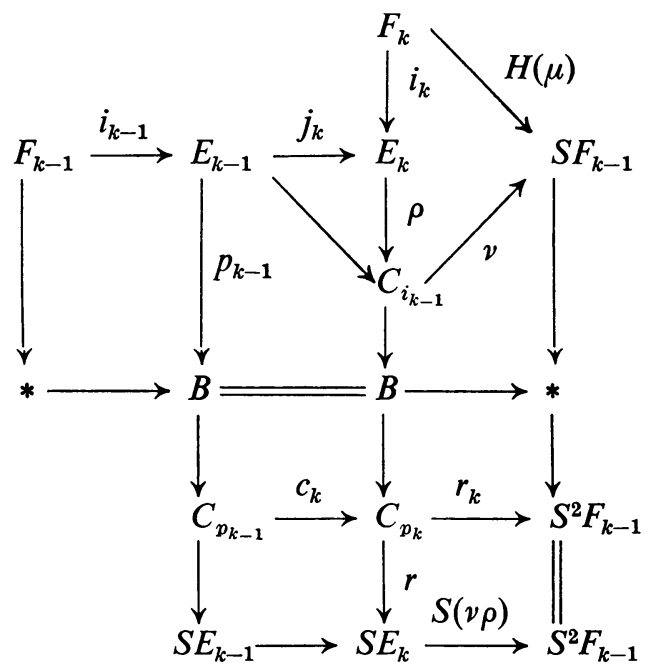

where $\rho$ is the homotopy equivalence given in the proof of Lemma 2.1 and $r_{k}$ is induced by $v \rho$. The following follows from the fact that $r \sigma_{k}=S i_{k}$.

LEMMA 2.5. $r_{k} \sigma_{k} \simeq S H(\mu)$.

We now proceed to the proof of Theorem 1.1. Consider the homology exact sequence

$$
\cdots \rightarrow H_{q}(E) \rightarrow H_{q}(B) \rightarrow H_{q}\left(C_{p}\right) \rightarrow H_{q-1}(E) \rightarrow \cdots \rightarrow H_{m}(B) \rightarrow 0
$$

obtained from the Puppe sequence for $p$. We shall show that the term $H_{q}\left(C_{p}\right)$ may be replaced in a certain range of dimensions by the algebraic one stated in the theorem.

By Lemma 2.3 we have that, for $m+1<q<n+1$,

$$
H_{q}\left(C_{p}\right) \cong H_{q}\left(C_{p_{1}}\right) \cong \cdots \cong H_{q}\left(C_{p_{q-m-2}}\right)
$$

and the sequence

$$
0 \longrightarrow H_{q}\left(C_{p_{q-m-2}}\right) \stackrel{c_{q-m-1 *}}{\longrightarrow} H_{q}\left(C_{p_{q-m-1}}\right) \longrightarrow H_{q-2}\left(F_{q-m-2}\right)
$$

is exact. Since $H_{k}\left(F_{q-m-1}\right)=0$ for $1 \leqq k \leqq q-2$, Lemma 1.1 of [5] gives rise to the following exact sequence

$$
\tilde{H}_{0}(\Omega B) \otimes H_{q-1}\left(F_{q-m-1}\right) \stackrel{\tilde{\mu}}{\longrightarrow} H_{q-1}\left(F_{q-m-1}\right) \stackrel{\sigma *}{\longrightarrow} H_{q}\left(C_{p_{q-m-1}}\right) \longrightarrow 0 .
$$


We see, using the lifting function of the Whitney join, that $\pi_{1}(B)$ acts on

$$
H_{k+m}\left(F_{k}\right) \cong \underset{(k \text { factors })}{(I \underset{\cdots}{\otimes}) \otimes} \otimes H_{m}(F)
$$

in such a way as described before Lemma 2.4. Hence

$$
\operatorname{Cok} \tilde{\mu} \cong \underset{(q-m-1 \text { factors })}{(I \otimes \cdots} \otimes \underset{Q}{\otimes} H_{m}(F)
$$

Combining these together yields the exact sequence

$$
0 \rightarrow H_{q}\left(C_{p}\right) \rightarrow \underset{(q-m-1 \text { factors })}{(I \otimes \cdots \otimes} \otimes{ }_{Q} H_{m}(F) \rightarrow \underset{(q-m-2 \text { factors })}{(I \otimes \cdots} \otimes H_{m}(F)
$$

for $m+1<q<n+1$. Thus Lemma 2.4 implies that

$$
H_{q}\left(C_{p}\right) \cong \operatorname{Tor}_{q-m-1}^{Q}\left(Z, H_{m}(F)\right) \text { for } m+1<q<n+1 .
$$

Note that, by Lemma 1.1 of [5], the sequence

$$
\tilde{H}_{0}(\Omega B) \otimes H_{m}(F) \rightarrow H_{m}(F) \rightarrow H_{m+1}\left(C_{p}\right) \rightarrow 0
$$

is exact, which shows that $H_{m+1}\left(C_{p}\right) \cong H_{m}(F) \otimes_{Q} Z$.

Finally let us write ${ }^{1} R=H_{n+1}\left(C_{p}\right)$ and $S=H_{n+2}\left(C_{p_{1}}\right)$. Then, by Lemma 2.3 , we have an exact sequence

and

$$
S \rightarrow H_{n}(F) \rightarrow R \rightarrow H_{n+1}\left(C_{p_{1}}\right) \rightarrow 0
$$

$$
H_{n+1}\left(C_{p_{1}}\right) \cong H_{n+1}\left(C_{p_{2}}\right) \cong \cdots \cong H_{n+1}\left(C_{p_{n-m-1}}\right)
$$

Also we have an exact sequence

$$
0 \rightarrow H_{n+1}\left(C_{p_{n-m-1}}\right) \rightarrow H_{n+1}\left(C_{p_{n-m}}\right) \rightarrow H_{n-1}\left(F_{n-m-1}\right) .
$$

Since the sequence

$$
\tilde{H}_{0}(\Omega B) \otimes H_{n}\left(F_{n-m}\right) \rightarrow H_{n}\left(F_{n-m}\right) \rightarrow H_{n+1}\left(C_{p_{n-m}}\right) \rightarrow 0
$$

is exact by Lemma 1.1 of [5], we see that Lemma 2.4 leads to

$$
H_{n+1}\left(C_{p_{1}}\right) \cong \operatorname{Tor}_{n-m}^{Q}\left(Z, H_{m}(F)\right) \text {. }
$$

Again from Lemma 2.3 we get exact sequences

and

$$
\begin{aligned}
& H_{n+1}(\Omega B * F) \rightarrow S \rightarrow H_{n+2}\left(C_{p_{2}}\right) \rightarrow 0, \\
0 \rightarrow & H_{n+2}\left(C_{p_{n-m}}\right) \rightarrow H_{n+2}\left(C_{p_{n-m+1}}\right) \rightarrow H_{n}\left(F_{n-m}\right)
\end{aligned}
$$

$$
H_{n+2}\left(C_{p_{2}}\right) \cong H_{n+2}\left(C_{p_{3}}\right) \cong \cdots \cong H_{n+2}\left(C_{p_{n-m}}\right) \text {. }
$$

${ }_{1}$ In the argument which follows, we assume $n>m+1$. In case $n=m+1$, some minor modifications will be necessary and the assertion of the theorem will turn out to be true. 
In addition Lemma 1.1 of [5] yields an exact sequence

$$
\tilde{H}_{0}(\Omega B) \otimes H_{n+1}\left(F_{n-m+1}\right) \rightarrow H_{n+1}\left(F_{n-m+1}\right) \rightarrow H_{n+2}\left(C_{p_{n-m+1}}\right) \rightarrow 0 .
$$

Hence we may infer from Lemma 2.4 that

$$
H_{n+2}\left(C_{p_{2}}\right) \cong \operatorname{Tor}_{n-m+1}^{Q}\left(Z, H_{m}(F)\right) \text {. }
$$

The commutativity follows from Lemma 2.5 and from the fact that the composite $S F_{\rightarrow}^{\sigma_{0}} C_{p} \rightarrow S E$ coincides with $S i$ and that $S F_{\stackrel{\sigma_{0}}{\rightarrow}}^{\rightarrow} C_{p} \stackrel{{ }_{1}}{\rightarrow} C_{p_{1}}$ is essentially the Puppe sequence of $\sigma_{0}$. This completes the proof of Theorem 1.1 .

\section{REFERENCES}

1. B. Eckmann and P. J. Hilton, Operators and cooperators in homotopy theory, Math. Ann. 141 (1960), 1-21. MR 22 \#5964.

2. B. Eckmann and U. Stammbach, On exact sequences in the homology of groups and algebras, Illinois J. Math. 14 (1970), 205-215. MR 42 \#4615.

3. T. Ganea, Homologie et extensions centrales de groupes, C. R. Acad. Sci. Paris Sér. A-B 266 (1968), A556-A558. MR 38 \#240.

4. Y. Nomura, The Whitney join and its dual, Osaka J. Math. 7 (1970), 353-373.

5. - Homology of a group extension, Pacific J. Math. (submitted).

6. J. Schmid, Zu den Reduktionssätzen in der homologischen Theorie der Gruppen, Arch. Math. 15 (1964), 28-32. MR 28 \#5100.

7. U. Stammbach, Anwendungen der Homologietheorie der Gruppen auf Zentralreihen und auf Invarianten von Präsentierungen, Math. Z. 94 (1966), 157-177. MR 34 \#1377.

Department of Mathematics, College of General Education, Osaka UniVERSITY, TOYONAKA, OSAKA 560, JAPAN 